\title{
Overactive bladder syndrome pharmacotherapy: future treatment options
}

\author{
Andrzej Wróbel \\ Second Department of Gynecology, Medical University of Lublin, Lublin, Poland
}

\begin{abstract}
Overactive bladder syndrome (OAB) is a lifestyle disease and its incidence increases with age. Although it is not a life-threatening disease, it is known to have a significant impact on the quality of life. The first-choice pharmacological treatment of $O A B$ is antimuscarinics. However, their limited clinical effectiveness and unsatisfactory tolerance profile, combined with the advancement of knowledge on the aetiopathogenesis of the disease, have inspired research on new pharmacotherapy options for OAB. Basic research has provided foundations for the development of new $O A B$ treatments, which seem very promising and can be applied in clinical practice. The mechanisms of the studied compounds are based on their effect on certain receptors and neurotransmitters that contribute to regulating the micturition reflex. These compounds are not only more receptor-specific as compared to currently used drugs, but also some of them are organ-specific. Some of such compounds have already passed the proof-of-concept stage of development and have the therapeutic potential to determine the future of OAB pharmacotherapy. This review focuses on the mechanisms of substances that are now undergoing pre-clinical and clinical tests and their effects on the micturition cycle, while also identifying opportunities for using them with specific groups of patients. Due to the fact that OAB is a disease of symptoms and its aetiopathogenesis is complex, it seems that modern treatment methods should be tailor-made and based on the pathophysiological mechanisms that induce disease symptoms, rather than only treating the symptoms by inhibiting the contractility of the urinary bladder.
\end{abstract}

Key words: overactive bladder syndrome, detrusor overactivity, pharmacotherapy.

\section{Introduction}

Overactive bladder syndrome (OAB) is a symptombased diagnosis defined by the International Continence Society as urinary urgency with or without urinary incontinence, usually with frequency and nocturia in the absence of local pathological lesions which could cause the aforementioned symptoms. The incidence of this condition ranges from $14 \%$ to $30.9 \%$ and increases with age. Therefore, it is considered a social disease with incidence almost equal to hypertension, bronchial asthma and heart diseases.

The majority of drugs currently used in $O A B$ therapy appear to have peripheral effects. They can be categorised as drugs whose primary objective is to reduce the contractility of the detrusor urinae muscle and to affect the sensory nerves. Antimuscarinics are considered the first-line therapy of OAB. However, the compliance and persistence of this group of medications is low. Their use is limited by their poor clinical effectiveness, which is sometimes unsatisfactory, and with well-known side effects.

These limitations of modern-day OAB pharmacotherapy have provided a stimulus for exploring the is- sue of the pathophysiology of this condition, which, in turn, has facilitated the development of new drug treatments. The effectiveness of $O A B$ treatment depends on the accurate identification of potential pharmacotherapy goals. Below there is a revised discussion of potential areas of operation and mechanisms that could become the basis for synthesising new effective substances in OAB pharmacotherapy.

\section{The serołonergic system}

Pre-clinical studies have shown that serotonin receptor agonists inhibit parasympathetic activity and facilitate sympathetic and somatic activity, while antagonists have opposite effects. The use of methysergide, a non-selective 5-HT receptor antagonist, or zatosetron, a $5-\mathrm{HT}_{3}$ receptor antagonist, leads to a reduced micturition threshold. Serotonin activity in the spinal cord seems to inhibit the detrusor function and facilitate the urethral sphincter function, which is mediated by the $5-\mathrm{HT}_{1 \mathrm{~A}}, 5-\mathrm{HT}_{2}$ and $5-\mathrm{HT}_{3}$ receptors [1]. The central serotonergic system is activated indirectly by the $5-\mathrm{HT}$ inhibitors of reuptake and directly by the $5-\mathrm{HT}_{1 \mathrm{~A}}$ and $5-\mathrm{HT}_{2}$ receptor agonists, and weakens the contractions 
of the detrusor and increases the threshold capacity of the bladder. Detrusor urinae has a high $5-\mathrm{HT}_{4}$ receptor density. Clinical studies have proven that cisapride, a selective $5-\mathrm{HT}_{4}$ receptor agonist, shows positive outcomes in patients with urinary retention following a spinal cord injury. In turn, the use of piboserod, a selective $5-\mathrm{HT}_{4}$ receptor agonist, has proven to inhibit detrusor hyperreflexia.

\section{The dopaminergic system}

The dopaminergic system can both inhibit and stimulate the micturition cycle by affecting, respectively, either $D_{1}$-like $\left(D_{1}\right.$ and $\left.D_{5}\right)$ receptors, which tonically inhibit the micturition reflex, or $D_{2}$-like $\left(D_{2}, D_{3}\right.$ and $\left.D_{4}\right)$ receptors, which pave the way for it. When this tonic, inhibiting effect of the dopaminergic system is blocked by destroying the nigrostriatal pathways, patients show motor symptoms that are similar to those observed in Parkinson's disease and OAB. Patients with Parkinson's disease have been found to be more likely to experience detrusor overactivity (DO), which seems to be a consequence of dopamine deficiency in the striatum structures and the insufficient activation of $D_{1}$-like inhibiting receptors. Detrusor overactivity symptoms in these patients are suppressed as a result of $D_{1}$ receptors being stimulated by selective agonists (pergolide). On the other hand, $D_{2}$ receptor stimulation with quinpirole or bromocriptine has proven to increase the reactivity of detrusor urinae. Cerebral infarction was found to cause increased $D_{2}$ receptor density and decreased $D_{1}$ receptor density, resulting in DO symptoms [2]. Therefore, it seems that selective $D_{2}$ receptor antagonists can be used in OAB pharmacotherapy in patients after cerebral infarction. The stimulation of central $D_{1}$-like dopamine receptors, with the simultaneous blocking of $D_{2}$ receptors, could prove to be an alternative of interest in $\mathrm{OAB}$ pharmacotherapy.

\section{Glutamic acid}

Acting as a neurotransmitter for both, the supraspinal efferent pathways and the afferent limb of the micturition reflex, glutamic acid affects the micturition cycle. Its increased concentration in Onuf's nucleus stimulates the pudendal nerve to release acetylcholine, which induces, via nicotinic receptors, contractions of the external sphincter muscle of the urethra.

Glutamic acid is a trigger that regulates the activity of the urethral sphincter, and 5-HT and NA determine the strength of the signal that reaches the sphincter. Without glutaminergic stimulation, increased $5-\mathrm{HT}$ and NA concentrations will not cause any changes in the activity of the urethral sphincter. However, with glutaminergic stimulation, 5-HT and NA will amplify the stimulating effect of glutamic acid, leading to increased contraction in the urethral sphincter.

Ionotropic glutamate receptors can be divided into three sub-types: NMDA, AMPA and kainate receptors. Detrusor overactivity symptoms visible in animals suffering from cerebral infarction caused by the closing of the middle cerebral artery (MCA) are accompanied by increased NMDA receptor density in the brainstem, and its reduced density in the forebrain. Detrusor overactivity symptoms in these animals were not visible when, before the MCA was closed, dizocilpine, a non-selective NMDA receptor antagonist, or a selective AMPA receptor antagonist, had been used [3]. The direct stimulation of glutamic acid receptors seems to be difficult to achieve. Moreover, it has been shown that agonists of these receptors can have hypertensive effects, which would constitute a major adverse effect of this group of compounds.

\section{Gamma-aminobutyric acid}

Gamma-aminobutyric acid (GABA) serves the role of an inhibiting neurotransmitter in central nervous system (CNS). GABA $A$ and $G_{A B A}$ receptors are involved in spinal and supraspinal mechanisms that control the micturition reflex. Positive treatment outcomes in bladder overactivity seem to be achieved by enhancing GABAergic transmission by stimulating $\mathrm{GABA}_{\mathrm{A}}$ and $G_{B B} A_{B}$ receptors, inhibiting GABA reuptake, or inhibiting GABA transaminase. It has been shown that the pontine micturition centre is under the tonic, inhibitory influence of the GABAergic system. The centre causes urinary bladder contractions by directly stimulating parasympathetic bladder motor neurons, while also inducing the relaxation of the external sphincter muscle of the urethra by stimulating GABAergic interneurons in the gray commissure of the spinal cord, which, in turn, inhibits the motor neurons of the external sphincter muscle of the urethra. Experimental studies have proven that the administration of exogenous GABA or muscimol, a $G_{A B A}$ receptor agonist, inhibits the detrusor urinae contraction reflex. These outcomes were reversed by administering bicuculline, a $\mathrm{GABA}_{\mathrm{A}}$ receptor antagonist. In patients with spinal cord injuries, the use of baclofen, a $G_{A B A}$ receptor agonist, has proven to reduce detrusor hyperreflexia [4]. The GABAergic system can potentially be of interest in OAB pharmacotherapy. $\mathrm{GABA}_{B}$ receptor agonists, such as baclofen, are used to treat detrusor hyperreflexia secondary to spinal cord injuries, also in patients with bladder spasticity.

Four different types of GABA transporters have been identified. Tiagabine is a selective inhibitor of one of them, GAT ${ }_{1}$. It has been shown that this drug inhibits the reuptake of GABA to increase the extracellular level of this neurotransmitter and to inhibit micturition. Tiagabine has been found to reduce micturition pres- 
sure and post-void residual volume, and to increase the volume of the urinary bladder.

\section{Ion channels}

In humans, the detrusor muscle includes at least two types of $\mathrm{K}^{+}$channels, namely ATP-sensitive channels $\left(\mathrm{K}_{\text {ATP }}\right)$ and calcium channels $\left(\mathrm{K}_{\mathrm{Ca}}\right)$. When these channels are opened, smooth muscles are hyperpolarised, which, in turn, inhibits $\mathrm{Ca}^{2+}$ channel opening, thus reducing the influx of calcium into the cell. Under physiological conditions, $\mathrm{K}_{\text {ATP }}$ channels are closed when involved in myorelaxant activity.

It has also been shown that $\mathrm{K}_{\mathrm{Ca}}$ (BK and SK) channels are important for bladder functioning. $\mathrm{BK}_{\mathrm{Ca}}$ and $\mathrm{SK}_{\mathrm{Ca}}$ channels play a significant role by being a part of the negative feedback that reduces the influx of extracellu$\operatorname{lar} \mathrm{Ca}^{2+}$, thus affecting the activity of the urinary bladder.

Compounds that open $\mathrm{K}^{+}$channels inhibit spontaneous bladder contractions without affecting micturition pressure [5]. Drugs that operate in this way can be useful in $O A B$ therapy by inhibiting detrusor hypersensitivity during the urine collection stage, but appear not to affect the micturition stage.

It has been established that nifedipine inhibits detrusor contractions in humans, which suggests that these contractions require the influx of extracellular $\mathrm{Ca}^{2+}$ through stretch-activated L-type $\mathrm{Ca}^{2+}$ channels.

The increased sensitivity of bladder neurons can be induced by changes in $\mathrm{Na}^{+}$channel expression. These channels are divided into tetrodotoxin-sensitive (TTX-S) and tetrodotoxin-resistant (TTX-R). Cystitis has been found to be accompanied by an increased TTX-R Na+ channel expression, and spinal cord injuries to be accompanied by changes in TTX-S $\mathrm{Na}^{+}$channel expression. It seems that the selective $\mathrm{Na}^{+}$channel block can be an effective OAB treatment method. Gabapentin is a sodium channel-blocking drug currently undergoing clinical trials.

\section{Prostanoids}

Prostaglandins modulate the afferent limb of the micturition reflex. Since changes in afferent impulse activity are considered one of the OAB causes, it seems that drugs that affect mechanisms involving prostaglandins can be of interest in the modern pharmacotherapy of this disease.

It has been shown that the use of non-selective cyclooxygenase (COX) inhibitors such as ketoprofen or indomethacin, causes a rise of the micturition threshold and the urinary bladder volume in detrusor hyperreflexia. Cyclooxygenase inhibitors reduce bladder-hyperactivity symptoms by blocking COX-2 activity and, consequently, inhibiting the stimulation of capsaicin-sensitive fibres. These are not recommended in haemorrhagic and interstitial cystitis, i.e. in conditions in which the epithelium of the urinary tract is not protected by prostaglandins. Indomethacin and ketoprofen improve the subjective symptoms of OAB but their impact on bladder functioning has not been confirmed in urodynamic testing. Beneficial effects on cystometric parameters have been demonstrated in relation to flurbiprofen, which have proved to reduce the frequency of urinary urgency and to increase bladder capacity. However, in view of the reports on the influence of selective COX-2 inhibitors on the cardiovascular system, the future of this research and development line is uncertain.

Therefore, desirable OAB treatment outcomes will be achievable without damaging the urinary bladder walls, perhaps not so much through the impact on COX as on specific prostanoid receptors. This statement is supported by research which has shown that animals which have been genetically deprived of the $\mathrm{EP}_{1}$ receptor do not show hyperreflexia symptoms following the intravesical administration of $\mathrm{PGE}_{2}$ [6].

\section{Tachykinin receptors}

Increased afferent impulsation plays an important role in $O A B$ aetiopathogenesis. Tachykinins serve as transmitters for sensory nerves. They operate via specific receptors. There are three types of tachykinin receptors: NK-1, NK-2 and NK-3. It has been shown that the contractions of human detrusor, induced by tachykinins, are only mediated by NK-2 receptors. The release of tachykinins from afferent nerve endings causes detrusor urinae hypersensitivity through NK-2 receptors, leading to a reduced micturition reflex threshold and the appearance of phasic contractions. The findings of a pre-clinical study which used NK-2 receptor antagonists allow the conclusion that these compounds can become a promising group of medications in OAB pharmacotherapy. Interestingly enough, it has been established that the use of Rho-kinase inhibitors leads to the inhibited contractile activity of the bladder, mediated by NK-2 receptors. NK-1 receptor antagonists also increase the micturition threshold, which suggests that tachykinins are involved in supraspinal mechanisms and makes NR receptors in the spinal cord a potential objective of new pharmaceuticals that could become effective in $O A B$ therapy. Urodynamic testing has proven that NK-2 receptor antagonists inhibit hyperreflexia in bacterial cystitis and in cases of a spinal cord injury, which suggests their possible application in patients suffering from such injuries or multiple sclerosis, which are often accompanied by OAB symptoms [7].

\section{TRPV ${ }_{1}$ receptors}

$\mathrm{TRPV}_{1}$ receptors are located on afferent neurons (mainly type $\mathrm{C}$, but also type $\mathrm{A} \delta$ ). Capsaicin and resiniferatoxin, their selective agonists, affect the sen- 
sory functions of the bladder, thus decreasing TRPV receptor density. A single administration of vanilloids causes the depolarisation of afferent fibres with TRPV receptors, and long-term exposure reduces sensitivity, disturbs the functioning, and completely destroys the peripheral nerve endings. With the aforementioned changes, vanilloids increase urinary bladder capacity in

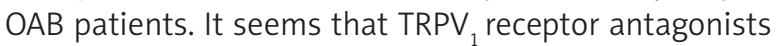
can become an alternative of interest in $\mathrm{OAB}$ pharmacotherapy. It has been demonstrated that they block ATP from being released from the urothelium as a result of urinary bladder walls' being stretched, and their use has proven not to produce adverse effects typical of $\mathrm{TRPV}_{1}$ agonists. The possibility of using TRPV receptor $_{1}$ blocking in $O A B$ pharmacotherapy is supported by the capsazepine-caused reduction of detrusor urinae contractions induced by the administration of cyclophosphamide [8].

\section{Opioid receptors}

Opioid receptors ( $\mu, \delta$ and $\kappa$ ) are located in a number of structures that play significant roles in micturition control. It has been shown that morphine can increase the cystometric capacity of urinary bladder and block detrusor contractions. Furthermore, the use of naloxone, a $\mu$-opioid receptor antagonist, has proven to stimulate micturition, which suggests that the tonic activation of $\mu$-opioid receptors has a suppressive effect on the micturition reflex. It has also been established that the epidural administration of morphine to patients with $O A B$ induced by spinal cord damage leads to a reduced sensitivity of detrusor urinae.

A drug that is widely used in pre-clinical studies is tramadol, a weak agonist of $\mu$-opioid receptors. Its mechanism of operation is of particular interest for $O A B$ treatment, as this drug is a racemic mixture, and its (+) enantiomers stimulate $\mu$-opioid receptors, and (-) enantiomers inhibit noradrenaline uptake. It has proven to increase urinary bladder capacity and threshold pressure by inhibiting DO induced by apomorphine and caused by brain ischaemia, which makes it an option of interest for the pharmacological treatment of OAB. Tramadol is further characterised by the broad difference between a dose which inhibits micturition and one which induces urinary retention, which results from the simultaneous inhibition of the serotonin and noradrenaline uptake.

Nociceptin, an endogenous agonist of $\mathrm{OP}_{4}$ opioid receptors, is now undergoing clinical trials. Its effectiveness has been demonstrated in relation to patients with neurogenic bladder incontinence. It affects both the central structures that control micturition and peripheral mechanisms, by inhibiting the activity of neurons with TRPV 1 receptors, which results in the reduced amplitude of terminal detrusor contractions. There- fore, it is likely that $\mathrm{OP}_{4}$-receptor agonists can become a pharmacological option of interest for the neurogenic bladder and an alternative for the intravesical instillation of vanilloids [9].

\section{Purinergic receptors}

ATP released from nerve endings in the urothelium affects specific (purinergic) receptors located in detrusor urinae and on afferent fibres. There are two families of purinergic receptors: $P_{2} X$ and $P_{2} Y$. So far, as many as 7 sub-types of $P_{2} X$, and 8 sub-types of $P_{2} Y$, have been identified.

When ATP is administered into the arterial vessels of the urinary bladder, afferent fibres are stimulated and the activity of the detrusor increases. On the other hand, the desensitisation of purinergic receptors or the use of suramin, their antagonist, inhibits the afferent impulse activity of the urinary bladder. Urodynamic testing has shown that the application of the purinergic receptor antagonist causes an increase in urinary bladder volume. A significant reduction in DO symptoms in animals without $P_{2} X_{2}$ and $P_{2} X_{3}$ receptors suggests that selective antagonists of these receptors can become useful in clinical practice. These animals have been found to exhibit reduced micturition frequency and increased bladder volume, which suggests that these receptors are connected with the functioning of bladder mechanoreceptors.

$\mathrm{P}_{2} \mathrm{X}_{1}$ and $\mathrm{P}_{2} \mathrm{X}_{3}$ receptors appear to play a fundamental role in the micturition reflex. When $\mathrm{P}_{2} \mathrm{X}_{3}$ receptors are blocked, the sensitivity threshold of the urinary bladder increases. High density in $\mathrm{P}_{2} \mathrm{X}_{3}$ receptors has been demonstrated in $\mathrm{IB}_{4}$ nociceptors. It has been further shown that when saporin, a cytotoxin that selectively destroys $\mathrm{IB}_{4}$ neurons, is administered at the level of $\mathrm{L}_{6}-\mathrm{S}_{1}$ of the spinal cord, it reduces bladder overactivity induced by ATP. Therefore, it seems that these fibres can become an objective of interest in OAB pharmacotherapy. Drugs that affect $P_{2} X$ receptors can become a very effective group of pharmaceuticals for patients suffering from neuropathy or elderly patients, due to their increased purinergic transmission [10].

\section{Oestrogen receptors}

The presence of oestrogen receptors (ER) has been demonstrated in the detrusor urinae, the urothelium, the urethra, and in the pelvic floor muscles. Oestrogen receptors also appear in the pelvic parasympathetic ganglionic neurons that supply the bladder, which proves that these hormones can modulate the release of acetylcholine (Ach). Post-menopause oestrogen deprivation leads to increased micturition frequency and reduced voided volume, and induces an increase in basal and stretch-induced Ach release. This results in 
symptoms characteristic of $\mathrm{OAB}$, such as urgency, frequency and nocturia.

It has been shown that oestrogens have $\mathrm{K}^{+}$channel opening and $\mathrm{Ca}^{2+}$ antagonist activities in the bladder, and can inhibit the rho kinase (ROCK) function in bladder detrusor without affecting ROCK expression. Oestrogen therapy directly affects the contractile activity of detrusor urinae by inhibiting the influx of calcium ions from the extracellular space into the cell and modifying the function of muscarinic receptors. It also reduces the amplitude and frequency of spontaneous rhythmic contractions, which are characteristic of detrusor instability, and the contractile response of the isolated detrusor muscle. Oestrogens modulate urogenital tissue perfusion and the cholinergic and adrenergic innervation of the bladder. It has been demonstrated that oestrogen replacement therapy can lead to an increased sensory threshold of the bladder.

The beneficial effects of oestrogens on OAB symptoms have been proven in clinical studies. It has been shown that the use of $17 \beta$-oestradiol vaginal tablets leads to a significant reduction in frequency, urgency and urge incontinence. Low doses of oestriadol or estriol administered intravaginally proved to reduce the frequency of nocturia and urge incontinence incidents, while also leading to an early first sensation to void and increased bladder capacity. Meta-analysis findings on oestrogen impact on OAB symptoms suggest that intravaginal replacement therapy is more effective than the systemic one. Moreover, another study has demonstrated that combination therapy using tolterodine and oestrogen cream increases the effectiveness of $O A B$ treatment in post-menopausal female patients [11].

\section{Cannabinoid receptors}

The central nervous system shows a high density of cannabinoid receptors $\left(C_{1}, C_{2}\right)$. The presence of $C B_{1}$, but not $C B_{2}$, receptors has also been demonstrated on dorsal root neurons. It has been established that $\mathrm{CB}_{1}$ receptor stimulation inhibits $\mathrm{DO}$ induced by the intravesical administration of NGF, this outcome being reversed by using its selective antagonist. Clinical trials have shown that cannabis sativa extract, which includes delta-9-tetrahydrocannabinol, administered sublingually, reduces $O A B$ symptoms in patients suffering from multiple sclerosis [12]. Therefore, it seems that $\mathrm{CB}_{1}$, and probably $\mathrm{CB}_{2}$, receptors can potentially be of interest to $\mathrm{OAB}$ pharmacotherapy.

\section{Kinin receptors}

Under physiological conditions only the $B_{2}$ receptor was found in the bladder, but in an infected bladder both kinin receptors $\left(B_{1}\right.$ and $\left.B_{2}\right)$ were expressed. The presence of $B_{1}$ receptors in an infected urothelium, and the fact that their stimulation induces the release of ATP, seem to prove that the participation of kinins in OAB pathogenesis can be mediated by ATP. It has been established that bradykinin, $a B_{2}$ receptor agonist, induces ATP release from the urothelium cells, thus causing the detrusor to contract, an outcome that is inhibited by capsaicin and COX inhibitors. $B_{1}$ receptor antagonists, in turn, have proven to inhibit the overactivity of the detrusor induced by the administration of cyclophosphamide, leading to a considerable improvement in urodynamic parameters [13].

\section{Phosphodiesterase inhibitors}

Phosphodiesterases (PDE) regulate CAMP and CGMP levels in cytosol. It has been demonstrated that increased CAMP and CGMP concentrations in the detrusor urinae induces a decrease in intracellular calcium levels, and, in consequence, leads to its relaxation. The human bladder has been found to include PDE 1, 3, 4, 5 and probably 7. PDE1 and PGE3 inhibition induces an increase in CAMP levels, while PDE5 inhibition induces an increase in CGMP. Clinical trials on the effectiveness of vinpocetine, a PDE1 inhibitor, in OAB pharmacotherapy, did not show any statistically significant effectiveness compared to the placebo [14]. Nevertheless, it seems that, due to their ability to increase the urinary bladder compliance, PDE inhibitors could possibly be used in combination therapy with muscarinic receptor antagonists, especially in patients suffering from neuropathy.

\section{Nerve-growth factor}

So far, two types of nerve-growth factor (NGF) receptors have been discovered, i.e. TrkA, with a high affinity, and p75, with a low affinity, with NGF. TrkA is located on capsaicin-resistant fibres covered with neurolemma, and its density increases during inflammation processes. On the other hand, the high expression of p75 receptors in the urothelium has been demonstrated in OAB patients. This proves that NGF can increase afferent impulse activity, and, in consequence, induce OAB by stimulating both TrkA and $p 75$ receptors. This is corroborated by study findings, which showed that the intravesical administration of NGF induces DO. These symptoms were reversed by NGF-neutralising antibodies, which also proved to inhibit overactivity induced by inflammation. This suggests that when TrkA receptors are blocked, the activity of capsaicin-sensitive neurons is reduced.

In addition to affecting TrkA and p75, NGF also modulates the functions of TRPV ${ }_{1}, N K$ and voltage-gated sodium channels (Nav 1.8, SNS, PN3). It has been established that the activation of TrkA by NGF produces the expression of TRPV ${ }_{1}$ receptors, which suggests that the use of selective TrkA antagonists would inhibit 
afferent impulse activity. Although TrkA and p75 receptor blocking seems like an alternative of interest in $O A B$ pharmacotherapy, it is important to bear in mind the influence of NGF on the trophic function of sympathetic neurons [15].

\section{Corticotropin-releasing factor}

It is a generally accepted fact that the hypothalamicpituitary-adrenal (HPA) axis plays a significant role in the aetiopathogenesis of both depression and OAB. Medical literature often uses the informal term uropsychiatry, which emphasises the close relationship between these conditions. It has been demonstrated that the corticotropin-releasing factor (CRF) reduces the afferent impulse activity threshold of the micturition reflex and increases the contractile activity of the detrusor urinae. Its effects are mediated by endogenous ligands for CRF-R1. Clinical trials have confirmed that the areas characterised by a high CRF-R1 density, such as Barrington's nucleus in brainstem amygdala, prefrontal cortex or hippocampus, play an important role both in response to stress factors and in controlling the micturition reflex. Corticotropin-releasing factor stimulates micturition, and selective CRF-R1 antagonists were found to reduce DO symptoms. Corticotropin-releasing factor also affects micturition at the spinal cord level. Indeed, it has been demonstrated that the administration of capsaicin, leading to the selective destruction of afferent C-fibres, also induces a decrease in CRF expression, which confirms its significant role in conducting afferent impulses from the bladder. Therefore, CRF-R1 seems to be an objective of interest in $\mathrm{OAB}$ treatment, especially in patients with depression as co-morbidity [16].

\section{$D_{3}$ vitamin receptors}

$D_{3}$ vitamin receptors have been found in the human bladder and urethra. They have been found both in the urothelial cells and in the detrusor smooth muscle cells. It has been proven that vitamin $D$ analogues can affect the contractile activity of the detrusor through the up-regulation of $\mathrm{Ca}^{2+}$ entry through L-type $\mathrm{Ca}^{2+}$ channels and by inhibiting ROCK signalling. Clinical trials have proven that the use of elocalcitol in patients with clinical OAB symptoms and the urodynamic diagnosis of DO leads to a reduced number of urinary incontinence episodes and the subjective improvement in patient perception of the bladder condition. However, no impact of this therapy has been found in relation to DO symptoms visible in urodynamic tests. The use of elocalcitol in patients with benign prostatic hyperplasia was demonstrated to result in reduced urgency and daytime and night-time frequency. On the other hand, patients with idiopathic overactive bladder who took elocalcitol experienced an increase in the bladder volume at the first desire to void and in the voided volume [17]. These findings suggest that vitamin $D$ is involved in mechanisms that control suburothelial afferent nerves, which, in turn, regulate detrusor activity.

\section{Rho-kinase inhibitors}

Rho-kinase (ROCK) is serine/threonine kinases. Rho-kinase impacts on the tone of the bladder detrusor through several mechanisms, which can increase myosin light chain (MLC) phosphorylation by MLC phosphatase phosphorylation (inactivation), modify actomyosin interaction by activating LIM-kinase, which promotes actin fibre formation by inactivating the actin-depolymerisation factor cofilin, and also directly phosphorylate MLC.

The role of ROCK in bladder contractility is not restricted to its links with $M$ receptors, as it was proven that it also has an impact on purinergic receptors and receptors for neurokinins and bradykinins. Rho-kinase pathways can change in pathological conditions such as bladder hypertrophy, hypertension and diabetes. It was demonstrated that ROCK inhibitors not only suppress bladder contractions induced by exogenous compounds, but also lower the basal tone of the bladder in the absence of stimulation, which can point to the role of the ROCK pathway in keeping the basal tone of the bladder. The evidence demonstrated that muscarinic acetylcholine receptor signal transduction pathway is linked to ROCK [18]. Due to the above, it appears that the pharmacological inhibition of the ROCK pathway leading to the inhibition of $\mathrm{Ca}^{2+}$ sensitisation and MLC phosphorylation can turn out to be a very effective option of the pharmacological treatment of OAB.

\section{$\beta_{3}$ adrenergic receptor agonists}

$\beta_{3}$ receptors are of crucial importance for the correct functioning of the bladder. When these are stimulated, adenylate cyclase is activated via a $G$ protein, which leads to an increase in the intracellular concentration of CAMP and the subsequent activation of CAMP-dependent protein kinase $A$. This kinase, in turn, phosphorylates myosin light chains responsible for inhibiting interactions between actin and calcium-calmodulin-dependent myosin. An increased CAMP level also reduces $\mathrm{Ca}^{2+}$ concentration in cytoplasm. The clinical outcome of these changes is a significant increase in the functional volume of the bladder, which decreases micturition frequency. An mRNA analysis has shown that $97 \%$ of $\beta$-adrenergic receptors in the human bladder are subtype $\beta_{3}$, and about $1.5 \%$ are sub-types $\beta_{1}$ and $\beta_{2}$ [19].

The detrusor muscle contracts when muscle contraction neurotransmitters (acetylcholine and ATP) are 
released, and, consequently, $\beta_{3}$ receptor agonists do not prevent bladder voiding. Although by blocking $M_{2}$ and $M_{3}$ receptors anti-muscarinic preparations inhibit involuntary detrusor contractions and can produce micturition disorders, $\beta_{3}$ receptor stimulation causes the bladder to relax during the urine collection stage, without affecting the micturition stage [20].

\section{Conclusions}

Previous pre-clinical studies have identified new objectives for OAB pharmacotherapy. Currently, clinical assessment is being carried out for organ-specific $M_{3}$ receptor antagonists, $\beta_{3}$ receptor agonists, potassium channel opening compounds, and Rho-kinase inhibitors. Other alternatives include substances that affect afferent impulse activity, such as $\mathrm{NK}_{2}, \mathrm{TRPV}_{1}$ and $\mathrm{P}_{2} \mathrm{X}$ receptor antagonists. The advancement of knowledge on $\mathrm{OAB}$ pathophysiology has also made it possible to influence the CNS centres that regulate the micturition cycle by affecting dopamine, serotonin, glutaminergic or CRF receptors. It seems that these new options of pharmacological OAB treatment will facilitate the customisation of treatment on the basis of aetiopathogenetic mechanisms that lead to the development of disease symptoms.

\section{Disclosure}

Author reports no conflicts of interest.

\section{References}

1. Espey MJ, Downie JW. Serotonergic modulation of bladder function before and after spinal transection. Eur J Pharmacol 1995; 287: 173-177.

2. Yoshimura N, de Groat WC. Neural control of the lower urinary tract. Int J Urol 1997; 4: 111-118.

3. Yotsuyanagi S, Yokoyama O, Komatsu K, Kodama K. Expression of neural plasticity related genes in the pontine tegmental area in rats with overac tive bladder following cerebral infarction. J Urol 2001; 166: 1148-1156.
4. Igawa Y, Mattiasson A, Andersson KE. Effects of GABA-receptor stimulation and blockade on micturition in normal rats and rats with bladder outflow obstruction. J Urol 1993; 150 (2 Pt 1): 537-542.

5. Shieh CC, Feng I, Bucknel SA. Functional implication of spare ATP-sensitive $\mathrm{K}(+)$ channels in bladder smooth muscle cells. Ex Pharmacol Ex Ther 2001; 296: 669-675.

6. Andersson KE, Weln AJ. Pharmacology of the lower urinary tract: basis for current and future treatments of urinary incontinence. Pharmacol Rev 2004; 56: 581-631

7. Lecci A, Maggi CA. Tachykinins as modulators of the micturition reflex in the central and peripheral nervous system. Regul Pept 2001; 101: 1-18.

8. Ost D. Topography of the vanilloid receptor in the human bladder: more than just nerve fibers. J Urol 2002; 168: 293-297.

9. Lecci A, Giuliani S, Meini S. Nociceptin and the micturition reflex. Peptides 2000; 21: 1007-1021.

10. Moore KH, Ray FR, Barden JA. Loss of purinergic P2X(3) and P2X(5) receptor innervation in human detrusor from adults with urge incontinence. J Neurosci 2001; 21: 166-172.

11. Cardoso L, Lose G, McClish D. A systematic review of the effects of estrogens for symptoms suggestive of overactive bladder. Acta Obstet Gynecol Scand 2004; 83: 892-897.

12. Brady CM. An open-label pilot study of cannabis-based extracts for bladder dysfunction in advanced muitiple selerosis. Mult Scler 2004; 10: 425-433.

13. Chopra B. Expression and function of bradykinin B1 and B2 receptors in normal and inflamed rat urinary bladder urothelium. J Physiol 2005; 562: 859-871.

14. Truss MC. Initial clinical experience with the selective phosphodiesterase-I isoenzyme inhibitor vinpocetine in the treatment of urge incontinence and low compliance bladder. World J Urol 2000; 18: 439-443.

15. Zhang YH, Nicol GD. NGF-mediated sensitization of the excitability of rat sensory neurons is prevented by a blocking antibody to the p75 neurotrophin receptor. Neurosci Lett 2004; 366: 187-192.

16. Klausner A. Corticotrophin Releasing Factor reduce the micturition threshold when administered at central, spinal and peripheral levels. J Urol 2004; 171: 453-459.

17. Crescioli C, Morelli AM, Adorini L, et al. Human bladder as a novel target for vitamin D receptor ligands. J Clin Endocrinol Metab 2005; 90 : 962-972.

18. Peters SL, Schmidt M, Michel MC. Rho kinase: a target for treating urinary bladder dysfunction? Trends Pharmacol Sci 2006; 27: 492-497.

19. Cerruto MA, Asimakopoulos AD, Artibani W, et al. Insight into new potential target for the treatment of overactive bladder and detrusor overactivity. Urol Int 2012; 89: 1-8.

20. Bhide A, Digesu GA, Fernado R, Khullar V. Mirabegron - a selective $\beta 3$-adrenoreceptor agonist for the treatment of overactive bladder. Res Rep Urol 2012; 4: 41-45. 